\title{
Smart Hard Hat Applied in the Electric Power Industry
}

\author{
Genesis Alvarez ${ }^{1}$, Garvin Casinillo ${ }^{1}$, Anthony Goich ${ }^{1}$ and Felipe Soares ${ }^{1}$
}

\author{
Mentors: Dr. Hari Kalva ${ }^{1}$, Vichate Ungvichian ${ }^{1}$, Dr. María M. Larrondo Petrie ${ }^{1}$ and Mark Forest ${ }^{2}$ \\ ${ }^{1}$ Florida Atlantic University, Boca Raton, FL, 33431, USA \\ \{genesisalvar2013, gcasinillo2015, agoich, fsoares2014, hari.kalva, ungvich, petrie\}@fau.edu \\ ${ }^{2}$ Florida Power \& Light, Boca Raton, FL, 33431, USA mark.forrest@fpl.com
}

\begin{abstract}
Safety concerns within the substation, such as fractures, burns and wasp stings caused in the field, are addressed with the proposed solution. Risks can be avoided through tracking and communication. This paper focuses on safety improvement and increasing work efficiency in the electric substations and an overview of the entire system and its functions are presented. The extension of the hard hat was designed on Tinkercad, the 3Dprinted ring can be placed on a typical hardhat. In sequel, the corresponding mobile application is created using the Xcode 8 program and Swift 3 programming language. Feedback from utilities workers is incorporated to modify the wireless technology. Overall, the S\&S Tractus incorporates six features which allow workers to complete their tasks safer and more efficiently than ever before. It provides early warnings of potential safety issues, making interdepartmental phone calls, speech recognition for note taking, a global positioning system (GPS), photo taking and headlamp adjustments.
\end{abstract}

Keywords: Smart hardhat, wearable devices, safety, GPS, Power utilities, smart grid technology

\section{INTRODUCTION}

Substations are key components in power transformation, protection and control in the power system network. Intelligent electronic devices (IEDs) are implemented within the substation and performs electrical protection functions and have monitoring capabilities that communicate directly to a SCADA system [1]. Substation automation uses data from IEDs to activate power switches, send measured data and overall facilitate the substation monitoring, control and protection. Significant factors within the substation are safety and work efficiency. The purpose of this paper is to reduce the amount of injuries and improve an employee's working process, benefiting not only the worker but the utility company itself. "OSHA has estimated that an average of 12,976 lost-workday injuries to and 86 fatalities of electric power generation, transmission, and distribution employees occur annually" [2]. Currently, employees must wear personal protective equipment when they are in the field, including flame resistant clothing, hard hats, safety glasses and gloves.
Typical causes of substation accidents occur due to communication errors, vehicles, barriers and boundaries. The name of the project is called Smart \& Safe Tractus (S\&S Tractus).This is a next generation device aimed to significantly improve worker safety in the power industry. It allows its users to record hazard logs with automated speech recognition, take photos of their current tasks, make phone calls, all with the push of a button and provides early warnings of potential safety issues. Also, a GPS feature is included within the device to locate employees in case of an emergency. A companion IOS mobile application enhances the capabilities of the S\&S Tractus with features such as user authentication, hazard documentation, access to substation diagrams and tutorial videos, and a phone call user interface. To construct the best solution the problem was divided into sub problems which were solved by constructing subsystems that would form the S\&S Tractus. The following subsystems were identified in Fig. 1:

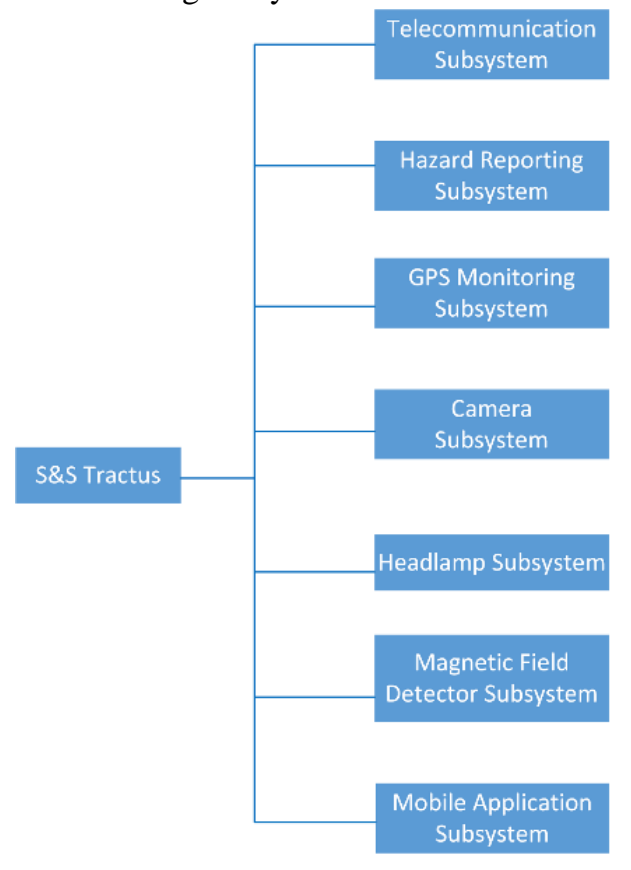

Fig. 1. System Diagram

Digital Object Identifier(DOI): http://dx.doi.org/10.18687/LACCEI2017.1.1.532 ISBN: 978-0-9993443-0-9

ISSN: $2414-6390$ 


\section{A. Magnetic Field Detector Subsystem}

This subsystem will warn the user if he/she is in the proximity of an object that emits a high magnetic field by illuminating an LED array.

\section{B. Hazard Reporting Subsystem}

This feature will allow the user to make a hazard report with a press of a button and upload the report to the Firebase database.

\section{Headlamp Subsystem}

This will illuminate the user's surrounding based on ambient lighting.

\section{Telecommunications Subsystem}

This subsystem will allow the user to make a phone call with a press of a button and a voice command.

\section{E. Camera Subsystem}

The camera will allow the user to take a picture and upload that image to the database.

\section{F. GPS Monitoring Subsystem}

This subsystem will monitor the user's GPS location every fifteen minutes and upload it to the database.

\section{G. Mobile Application Subsystem}

This iOS mobile application will run on a tablet and queries the Firebase database to allow the user to access substation information, substation documents, a contact list and tutorial videos after authentication.

\section{SYSTEM OVERVIEW}

The overview system is designed in Tinkercad, as shown in Fig. 2. The actual product constructed is shown in Fig. 3.

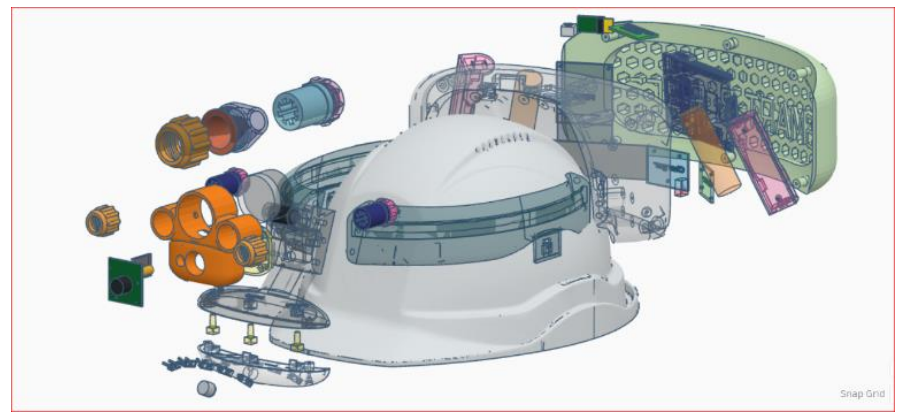

Fig. 2. CAD over view of product

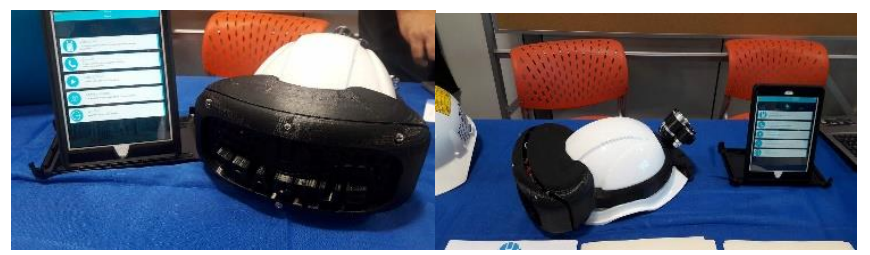

Fig. 3. Smart \& Safe Tractus

The Smart \& Safe Tractus is a product composed of multiple interdependent subsystems which include:

\section{A. Magnetic Field Detector Subsystem}

This feature is implemented as a hands free subsystem and will not have to be activated or deactivated by the user. This subsystem will turn on red warning LED's on the brim of the hard hat once it determines that the user is in close proximity to a high voltage object and will turn off when the user is located in a safe distance away from the high voltage object. The intensity of the magnetic field is dependent on the distance [2].

$$
H(d)=\frac{I N r^{2}}{2 \sqrt{\left(r^{2}+d^{2}\right)^{3}}}
$$

The current, number of turns of the coil and the radius of the coil is denoted as $\mathrm{I}, \mathrm{N}$ and $\mathrm{r}$.

\section{B. Hazard Reporting Subsystem}

The hazard reporting subsystem is aimed towards documenting an instance of a hazard as reported by the user. This feature will be activated when the user presses a designated button located on the brim of the hard hat and will give the user 15 seconds to record a message, which will be transcribed automatically to text and sent to the database.

\section{Headlamp Subsystem}

This will proved a semi hands-free subsystem. A button can be pushed to turn the headlamp on or off, once pressed the headlamp will adjust its brightness depending on how much illumination is needed.

\section{Telecommunications Subsystem}

This subsystem offers a semi hands-free interaction with the system to make a phone call. The user simply has to push a button and issue a voice command (e.g. "Call Anthony") and the phone call will be placed. The call button can be pressed anytime during the phone call if the user wishes to finish the call. This will allow workers to interact with other departments more effectively.

\section{E. Camera Subsystem}

This feature is intended to supplement the telecommunications subsystem by allowing the user to take pictures upon pressing a designated button on the brim of the hard hat. Once the button is pressed a laser beam will assist the user in aiming the camera and pressing the button a second time will take the picture. The picture that was taken can be viewed by an offsite personnel.

\section{F. GPS Monitoring Subsystem}

The GPS coordinate broadcasting subsystem was created with the intention of fortifying the safety of the user. This subsystem records and broadcasts the GPS coordinates of the user every 15 minutes and sends that information to a remote database that can be accessed by senior personnel in case of emergency [3].

\section{G. Mobile Application Subsystem}

This subsystem is an iOS mobile application and its features are implemented to act as a supplement to the S\&S Tractus. These 
features include, substation directions, user authentication, substation information, contact list and tutorial videos.

Due to the modular design approach of the S\&S Tractus the interaction between subsystems are kept to a bare minimum. The only interaction that the subsystems have is that they are being controlled centrally by the Raspberry PI microcontroller.

\section{SUBSYSTEM DESCRIPTION}

The telecommunication subsystem that displayed in Fig. 4 is activated when the phone call button is pressed by the user. Once the call button is pressed an interrupt will be sent to the Raspberry PI which in turn will run a python script that will turn on the microphone enabling the user to issue a voice command (e.g. "Call Genesis"). The python program will transcribe the user's voice command to text via Google speech to text API, that transcription will be used by the program to extract the contacts name and it will dial the contact's number from a list pulled from the database. Once a match is determined the program will start the phone call. The user can end the call at any time by pressing the call button. This subsystem meets usability requirement one which outlines the architecture of the communication system.
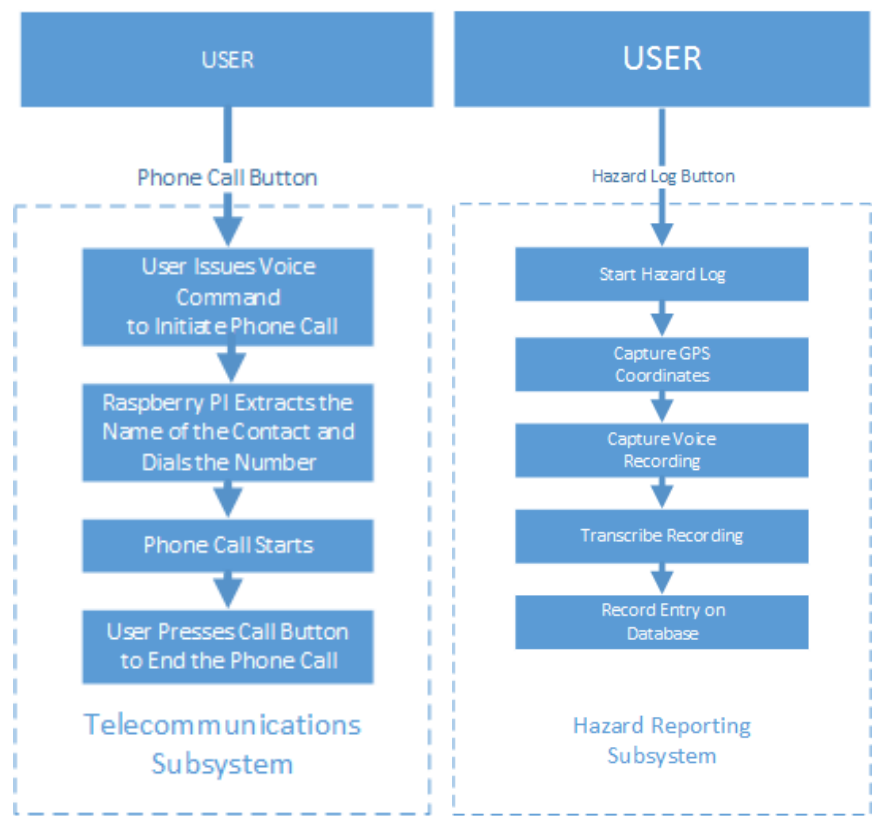

Fig. 4. Telecommunication and hazard reporting subsystem diagram

This hazard reporting subsystem is activated when the hazard button is pressed by the user. Once the call button is pressed an interrupt will be sent to the Raspberry PI which in turn will run a python script that will turn on the microphone enabling the user to verbalize the hazard. The user will have a maximum of 15 seconds to speak into the microphone before it cuts the user off. Once the voice has been transcribed to text the Raspberry PI will send the users report along with the date and time that the report was made. This subsystem will fulfill functional requirement three which states "The system shall record worksite hazard reports from the user via microphone and store the report along with GPS coordinates."

As shown on Fig. 5 the camera subsystem will be activated when the user presses the camera button. Once the button is pressed an interrupt will be sent to the Raspberry PI and a python script will run and turn on a laser beam that will assist the user in aiming the camera. When the user presses the button a second time a python script will turn on the camera and take a picture. That image will be saved to the Raspberry PI's local storage with the filename of the date and time the picture was taken. After the file is saved the python script will send that picture to the database. This subsystem partially fulfills functional requirement three and usability requirement one as it acts a supplement to both the hazard reporting system and the telecommunications system.

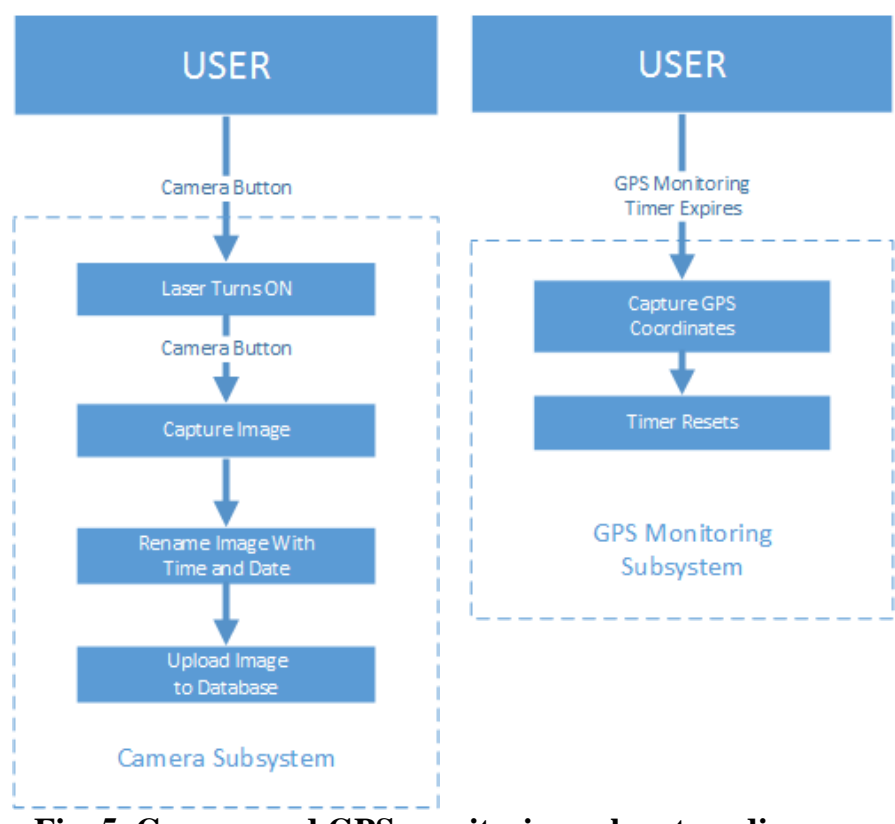

Fig. 5. Camera and GPS monitoring subsystem diagram

The GPS monitoring subsystem records and broadcasts the GPS coordinates of the user every 15 minutes and sends that information to the database. The GPS monitoring timer is set by a python program that runs on the background of the Raspberry PI. Once that timer expires that program will send an interrupt to the Raspberry PI which in turn will run a python script that will capture and send the user GPS coordinates to the database. Once this python script finishes running it will reset the GPS monitoring timer. This subsystem meets functional requirement two which states that "The system shall periodically record and transmit the user's GPS coordinates."

The headlamp subsystem, shown in fig. 6, will be activated when the user presses the headlamp button. Once the button is pressed the headlamp will turn on and adjust its brightness according to ambient lighting. The user can press the headlamp button again to turn it off. 
The magnetic field detector subsystem will only be activated when the user encounters an object that emits a high magnetic field. The system will continually get readings from the magnetic field sensor and will use that to calculate the user's proximity to an object that emits a high magnetic field. When the system calculates that the user is too close to that object it will turn on the LED array and when the user steps away from the threshold distance then it will turn off the LED array.

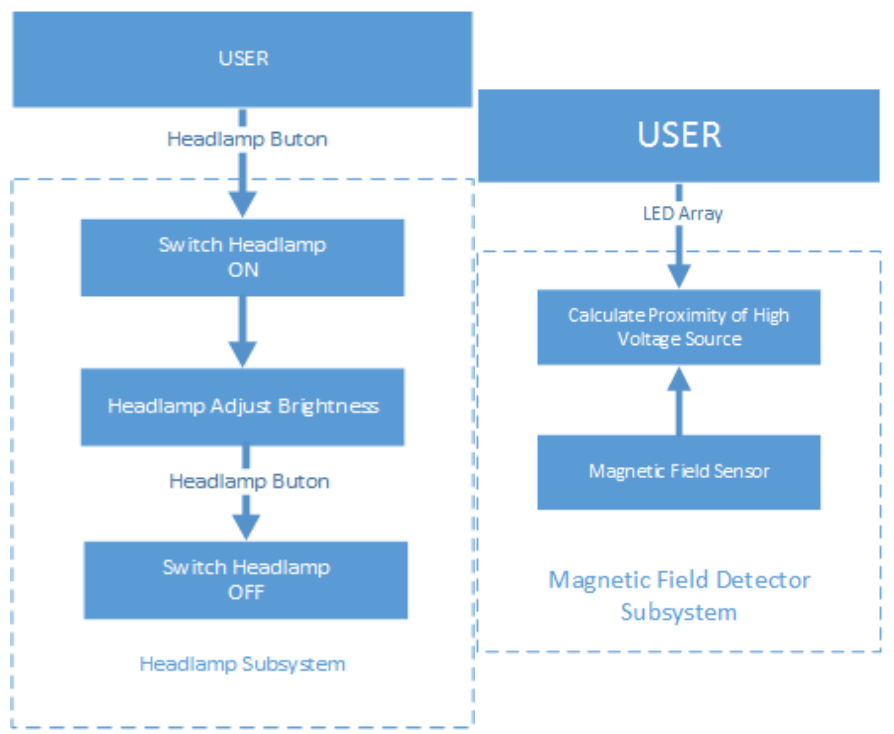

Fig. 6. Headlamp and magnetic field detector subsystem

\section{MOBILE APPLICATION}

This subsystem is an iOS mobile application running natively on an Ipad mini and its features are implemented to act as a supplement, see Fig. 7. These features include:

- User authentication

This will allow the user to log in .The user will not be able to access any of the other features of the application if he/she is not logged in.

- Substation Information

This features shows detailed information about a specific substation. Upon tapping the Substations button he/she will be presented with a list to view of all the substations. The user can then search for a substation by name or address and tap the substation he/she is interested in. Once the substation button has been pressed all the relevant information about that substation will be presented. (e.g. driving directions, hazard logs and relevant substation documents.

- $\quad$ Contact List

This will enable the user to view contacts that are approved by senior staff. The contact list is also searchable so that the user can for specific contacts either by first or last name or by phone number.

- Tutorial Videos

The portion allows the user to view tutorial videos that will orient them on the various company rules and regulations. The videos featured on the application will mostly contain

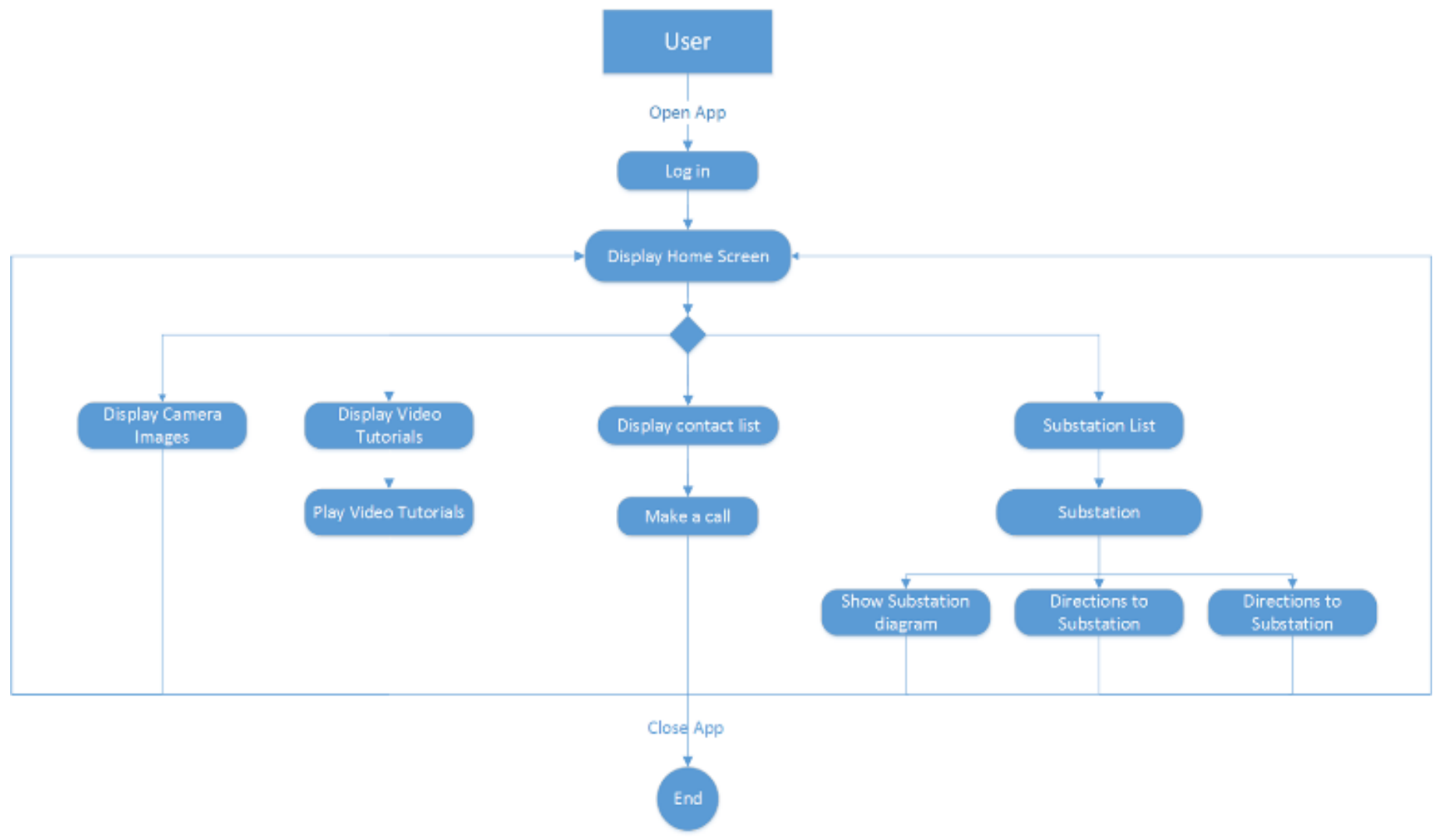

Fig.7. IOS mobile application subsystem 
material that pertain to worker safety around the workplace and background information on certain equipment such as the feeder breakers and micro controller relays within the substation. The user can access this feature by tapping on the Tutorial Videos button which will present the user with a list to view of all the tutorial videos. The user simply has to tap on a specific video and the video will play. The user can also search for a specific video if he/she wishes.

- Camera Image View

This segment will enable the user to view all the pictures taken by the camera. These images will be sorted by the date that they were taken.

\section{DATA COMMUNICATION}

The communication subsystem, shown in Fig. 8, is an integral part of the S\&S Tractus as it maintains the integrity of the information that is used by the user. This subsystem employs a one directional data stream structure as to maintain a strict security protocol. The essential component that can send data is the Raspberry PI, therefore it is the only component that can alter the database. The Raspberry PI sends data pertaining to hazard logs and the user's GPS location. The centerpiece of the communications subsystem is the Firebase database as it functions as a data bank for any information logged by the communications subsystem. The only component in the subsystem that can retrieve data from the database is the S\&S mobile application. It displays the substation information (name, address, hazard logs, etc.), contact list, tutorial videos and pictures taken by the camera.

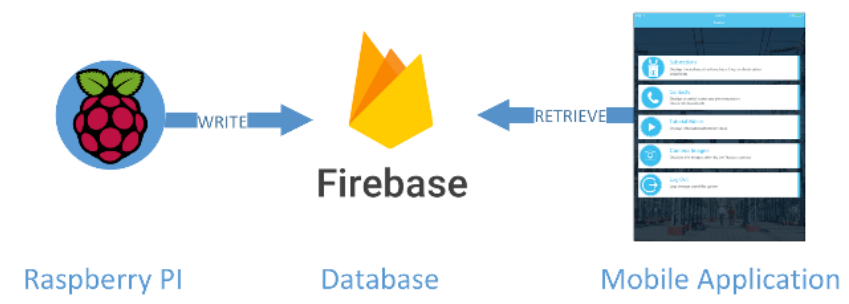

Fig.8. Data communication diagram

\section{MECHANICAL AND ELECTRICAL DESIGN}

The overall design of the S\&S Tractus is centered on the idea of maintaining the integrity of an existing hard hat while adding features that would help its users while they perform their daily tasks. In order to do this we opted for a modular design with a 3D printed device that would fit over any hard hat, shown in Fig. 9. Mechanical components such as tactile switches (call button, hazard button etc.) are located on the front brim of the Tractus towards the front of the hardhat. The camera and the headlamp are mounted on a swivel base, which allows the user to position the camera or the headlamp. The electrical components which include a printed circuit board, raspberry pi, Universal Serial Bus (USB) camera, aiming laser, batteries are all encased in $3 \mathrm{D}$ printed enclosures with proper spacing to ensure that all components suitably vented.
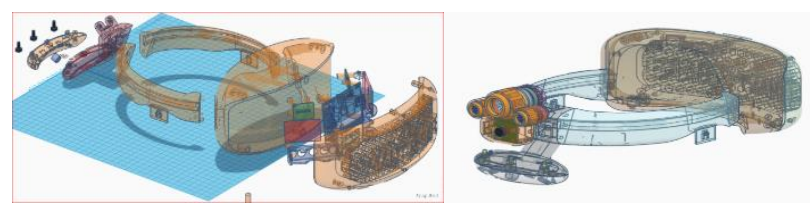

Fig.9. Front and back CAD image

The hardware components that were integrated in the S\&S Tractus were:

- $\quad$ Adafruit FONA808 module

- Raspberry Pi 3

- Tactile switch

- Cree's LED

- Web Camera(Webcam)

- Magnetic field sensor (DRV425 Module)

- Adafruit LED Sequins

- $\quad$ Printed Circuit Board (PCB)

- $\mathrm{DC} / \mathrm{DC}$ converter

\section{A. Adafruit FONA808}

This component will enable our system to track the GPS location of the user and make phone calls [4].This component was tested and calibrated by using the Raspberry Pi 3. We ran a sample program that enabled the FONA to make phone calls as well as transmit current GPS coordinates. We tested the FONA through both the Raspberry PI's USB and serial connection and we were able to duplicate the calling and GPS Transmission features [5].This component was integrated into the telecommunications subsystems to implement a phone call feature as well as the hazard reporting and the GPS monitoring subsystems to enable both systems to capture the user's GPS coordinates, as shown in Fig. 10.

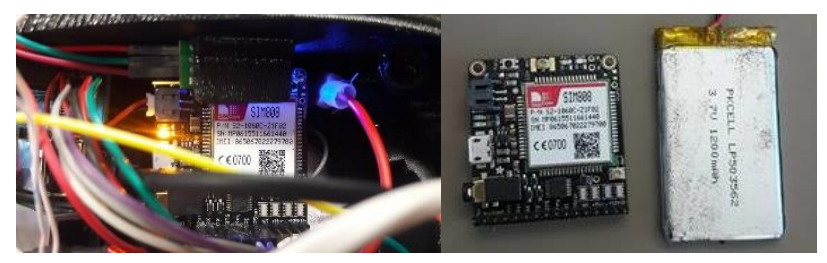

Fig.10. Adafruit FONA808 module

\section{B. Raspberry Pi 3}

This component is a single board computer and serves as the main controller of the system and manages all their functionalities. This component was tested and calibrated using the Raspbian operating system which is the most recent OS for this device.

\section{Tactile Switch}

This component is a button type switch that is optimal for printed circuit board mounting. This component interacts with the raspberry pi and it will function as the on/off switch for the headlamp, hazard log, phone call and the camera. 


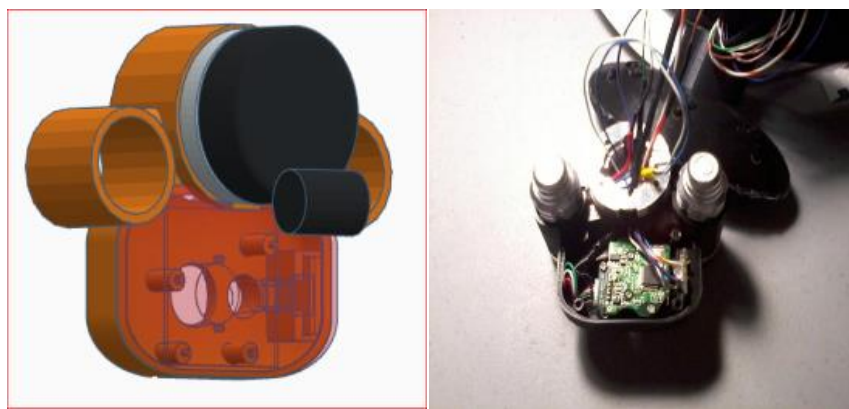

Fig. 11. Raspberry Pi camera

\section{Cree LED's}

This component consists of three high powered LED's. This component will provide the necessary illumination for the user once the tactile switch is turned on and turns off when the tactile switch is pressed a second time, see Fig. 11.

\section{E. Webcam}

In Fig. 11, the web camera uses a USB port and is located right below the center LED. A program that would transcribe voice to text using Google's speech to text API was implemented .This component will be integrated into the hazard reporting subsystem as well as the telecommunications subsystem since the user can use it to issue voice command and take pictures.

\section{F. Magnetic Field sensor}

This component is capable of accurately measuring magnetic fields. This component was integrated into the magnetic field detector subsystem where it continually reads the surrounding area for an object that emits a high magnetic field [6], as show in Fig. 12.

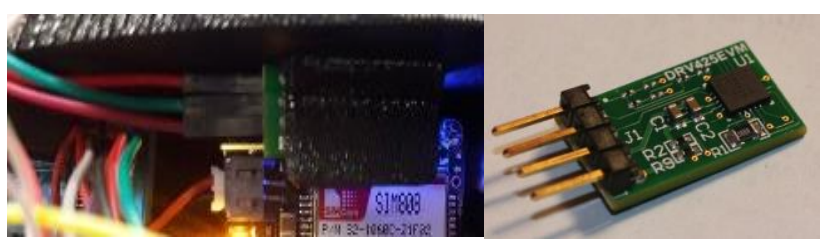

Fig.12. Magnetic field sensor

\section{G. Warning LED's}

This component will serve as a warning LED which will be turned on when a high voltage object is detected [7] This component was integrated into the magnetic field detector subsystem where it will light up when the magnetic field sensor senses an object that emits a high magnetic field, as shown in Fig. 13.
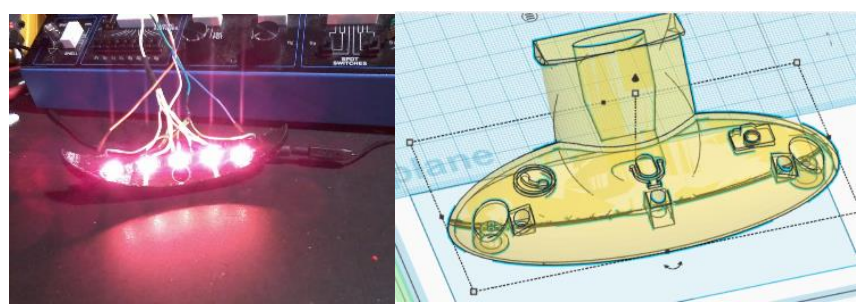

Fig.13. Adafruit LED sequins

\section{H. Printed Circuit Board}

The PCB encompasses the three tactile switch circuits, the laser for the camera subsystem and the magnetic field sensor circuit. The PCB was created using Eagle software, as displayed in Fig. 14. For the laser circuit, a pull down resistor and a NPN bipolar junction transistor (BJT) are included to allow the user to press the button and turn on the laser. The magnetic field circuit uses a comparator circuit to turn on the alert system at a designed distance.
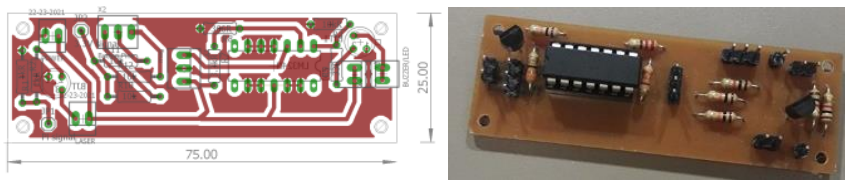

Fig.14. PCB and components placement

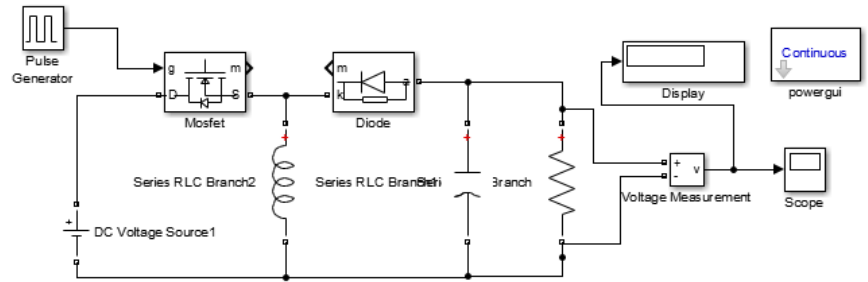

Fig. 15. DC/DC Buck-Booster Converter

I. DC/DC Buck booster converter

The voltage can increase or decrease depending on the load demand as shown in figure 15. The DC/DC buck-booster converter is utilized to step up the initial battery voltage from $3.7 \mathrm{~V}$ to $5 \mathrm{~V}$.

\section{TEsting AND PeRformance Evaluation}

In order to properly test the $S \& S$ Tractus, a subsystem testing procedure was engineered. This was done in order to properly vet each feature so that each failure will be isolated to that specific subsystem and will not affect the system as a whole. For examples of all the programs utilized, see [8].The testing and performance of each system is included:

\section{A. High Voltage Detector Subsystem}

This subsystem was tested by using a high powered magnet strategically placed from a distance ranging from a two to three inches from the S\&S Tractus. When the magnet got within a feet of the magnetic field sensor the red LED's illuminated and a warning speaker beeped. 


\section{B. GPS Tracking Subsystem}

This subsystem was tested in two parts:

First Test - this test involved testing the program that would send the user's GPS data to the database and present it in google map type application. This was done solely by using dummy GPS coordinates and writing that to the database and presenting those coordinates in a google map web application.

Second Test - the second portion of the test was testing the GPS coordinates being read from the FONA, this was done by running a program that would print GPS values to the terminal every 60 seconds. After passing this test the time it takes to print the value by 60 seconds was incremented until we got the program to print the GPS coordinates captured by the FONA every fifteen minutes. After passing these two tests we were able to integrate these two features and have this subsystem report the user's GPS coordinates to a google maps web application every fifteen minutes.

\section{Headlamp Subsystem}

The testing involved continually turning the illumination LED's on and off using the headlamp button.

\section{Hazard Detection Subsystem}

This subsystem was tested in two parts:

First Test - the program to write the hazard report as a string value to a database was created. This program also contained an algorithm to cross reference the user GPS location to a specific substation. This test was conducted by using a dummy string values to be written to the database as well as dummy GPS values.

Second Test - this test involved a program that would transcribe voice to text using Google's speech to text API. A program script was ran to test the program and the microphone. The program was tested by speaking into the microphone and viewing the words properly transcribed [9].

\section{E. Camera Subsystem}

The first test included creating a python program that takes a picture and sends it to the database was created. To test this program the camera was pointed at a certain direction and the program was ran to see if the image that we captured was clear. The image was properly uploaded to the database. The second test involved the laser that would help aim the camera for the user. The laser was tested using a power source, the PCB and multimeter to check whether or not voltage, current and resistance values coincided with the tolerance values of the lasers specification sheet.

\section{F. Telecommunications Subsystem}

This subsystem was tested in three parts:

First Test - this test involved a program that would transcribe voice to text using Google's speech to text API.

Second Test - a python program was created to retrieve all of the contacts from the database and cross reference that with a string value that would be replaced by the voice command extracted from Google's Speech to Text.
Third Test - the third test involved the FONA which is in charge of the phone call functionality for the system and was tested by using the Raspberry Pi 3.A sample program was ran that enabled the FONA to make phone calls and tested it through the Raspberry PI's USB and serial connection.

\section{G. IOS Mobile Application subsystem}

The IOS application was verified by scrutinizing its capability to read and write from the Firebase database. Each test coincided with a specific functionality:

Showing Substation List - Tested if all of the substations from the database were showing on the app. Substations locations were added and deleted in the database. The mobile application was verified (e.g. Address, documents, directions, hazard $\operatorname{logs}$ ) to see if those changes coincided with the database.

Display Contact List - Tested if all of the contact from the contact lists contained in the database were showing on the application.

Display and Play Video Tutorials - Tested if all of the links from the video tutorial section of the database were showing on the app and were playing properly.

Display Camera Images - Tested by taking pictures on the camera and checking whether those images showed properly on the app. Also, zooming capabilities were also verified.

\section{CONCLUSION AND FUTURE WORK}

In this paper, it is proposed to utilize wireless technology and a companion mobile application to develop a safer work environment and enhance the current work efficiency within the substation. The features such as wireless communication and speech recognition within this device can be customized base on users' occupation. The emergency medical services, fire rescue and the police department can benefit from this device as well. In the future a different method to sense high voltage environments and use a video stream method of communication between the departments will be established. 


\section{References}

[1] L. Andersson, C. Brunner and F. Engler, "Substation automation based on IEC 61850 with new process-close technologies," in 2003 IEEE Bologna Power Tech Conference Proceedings, , 2003

[2] Electric Power Generation, Transmission, and Distribution; Electrical Protective Equipment 59:4320-4476 | Occupational Safety and Health Administration.

[3] X. Lu, P. Wang, D. Niyato, D. I. Kim and Z. Han, "Wireless Charging Technologies: Fundamentals, Standards, and Network Applications," IEEE Communications Surveys Tutorials, vol. 18, pp. 14131452, Secondquarter 2016.

[4] M. E. Orientale, R. M. Boder and A. Marcos, Hard hat with additional technical features, Google Patents, 2014.

[5] Lady Ada, "Adafruit FONA Cellular + GPS Breakout," Adafruit Industries, Sep. 2016.

[6] S. S. W. Solutions, "Microsoft Word - SIM800 SeriesAT Command ManualV1.09.doc," 2015

[7] SLOU410A, "DRV425 Evaluation Module," Texas Instruments Incorporated, Sep. 2015.

[8] Wang, "1.10mm Height 1206 Package Pink Chip LED Technical Data Sheet," Lucky Light Electronics Co., Ltd., Dec. 2006.

[9] G. C. Felipe Soares, SS-Hardhat. Github, 2017

[10] Speech API - Speech Recognition | Google Cloud Platform. 
